\title{
Matched Filtration of LFM Signals with Non-Zero Initial Frequency
}

\author{
T. LESZCZYNSKI* \\ University of Technology and Life Sciences, S. Kaliskiego 7, 85-796 Bydgoszcz, Poland
}

\begin{abstract}
This paper presents the results of the research considering the influence of the initial frequency on linear frequency modulation signal compression. The signal compression has been performed using the digital matched filtration in the time domain. The ratio of peak to side lobe ratio with the width of the main lobe equaling one sampling period is the measure of the quality of compression. In general, a lower value of peak to side lobe ratio is obtained for linear frequency modulation signals with a non-zero initial frequency than with a zero initial frequency. The peak to side lobe ratio is systematically decreasing with the increase of the initial frequency $f_{1}$. The existing methods of band signals sampling, where the spectrum of the signals is transferred by means of the carrier signal $f_{0}$ of a significantly higher frequency than the band $B\left(f_{0} \gg B\right)$ may be effectively used for sampling signals considered in this study, which do not have a carrier. As a result, for the linear frequency modulation signals with a non-zero initial frequency $f_{1}=n B$ and a final frequency $f_{2}=(n+1) B, n=1,2, \ldots$, the same peak to side lobe ratio values will be obtained as for linear frequency modulation signals with a zero initial frequency and the identical band and time duration. The results of this research could be implemented in sonar and radiolocation techniques and medicine.
\end{abstract}

DOI: $10.12693 /$ APhysPolA.124.494

PACS: 84.40.Xb, 84.40.Ua

\section{Introduction}

An important feature of broadband signals with a linear frequency modulation (LFM) is their autocorrelation function. This function is characterized by the coefficient of a high value determining the ratio of the level of the main lobe to the maximal side lobe. The results of the correlation analysis significantly depend on the duration of a signal and, among others, the influence on the resolution of recognizing time shifts. During detections from the signal noises of a desired configuration, e.g. LFM signals, matched filtering, which is a kind of correlation analysis, is used. Applying matched filtering for these signals results we obtain their compression [1-4]. The measure of the compression quality is a peak to side lobe ratio (PSR) coefficient defined as logarithm of the quotient of the maximal value of the main lobe to the maximal value of the side lobe expressed in $\mathrm{dB}$.

Thus,

$$
\mathrm{PSR}=20 \log y_{\mathrm{c}} / y_{i},
$$

where $y_{\mathrm{c}}$ is the central sample of the main lobe (the result of a central convolution operation), $y_{i}$ is the maximal value of the convolution result appointed from other convolutions which form the side lobes.

A high PSR value makes it possible to detect and identify signals of this type, especially in the presence of significant noises. The resolution of detection of such signals is related with the accuracy of defining the main lobe position in time in an output signal of a matched filter (MF). Therefore the ratio of PSR with the width of the

\footnotetext{
*e-mail: leszcz@utp.edu.pl
}

main lobe equaling one sampling period is the measure of the quality of compression.

Usually, for chirp signal compression, "fast convolutions" in the frequency domain on the basis of a fast Fourier transform (FFT) and inverse FFT (IFFT) are used [3]. However, such an approach requires about $N \log _{2} N$ operations for $N$ samples of the chirp, the number of which limits the recognition speed, as well as the use of this method in fast-acting location systems. On the other hand, in this case the main lobe always consists of a few samples, and not one that limits recognition resolution. This is connected with the fact that the FFT output terms are multiplied by the frequency response (FR) terms and, afterwards, squared, added up, square-rooted and, afterwards, are subjected to IFFT [3]. As a result, close to the central sample, squared negative values always come together, and the main lobe consists of a few samples which worsen the resolution.

Therefore, we consider the optimal way to compress single short chirp signals with small bandwidth-time product (BT) to be the use of digital matched filtering on the convolutions in the time domain, on the basis of direct parallel algorithms. An $N$-channel parallel filter with one multiplier in each channel computes $N$ convolution results within $N$ periods $T_{\mathrm{S}}$ and this is $\log _{2} N$ times faster than the MF on "fast convolutions" if a sequential algorithm of FFT is used. In that parallel structure the non-linear operations on convolution results can easily be fulfilled.

In the hypothetical case of an $N$-channel parallel FFT/ IFFT processor, which allows us to obtain a result near to the direct parallel algorithm, it is also possible to realize non-linear operations on the results of each channel. Furthermore, the FFT result must be multiplied by a fil- 
ter FR, which corresponds to the impulse response (IR), and, afterwards, subjected to IFFT. In this way we obtain the result of matched filtering by $2 \log _{2} N+1$ simultaneous multiplication operations in each channel, which are realized only after $N$ sampling periods $T_{\mathrm{s}}$, which are necessary to enter all samples of a signal to a processor memory. Assuming that the duration of the multiplication plus addition operations does not exceed the sampling period $T_{\mathrm{s}}$ (in this context - time or clock period), the result of processing on the basis of the parallel FFT/ IFFT algorithm needs about $N+2 \log _{2} N+1$ clock periods which is longer by $2 \log _{2} N+1$ than the aforementioned direct parallel algorithm. Apart from that, each channel of the FFT parallel processor contains $\log _{2} N$ multipliers plus a complementary one to multiply a FFT result by the FR component. In contrast to this, the processor on direct convolutions has only one multiplier at each channel.

\section{Matched filtering algorithm}

To implement the digital matched filter, the input signal is represented in the form of a time series $\left\{x_{n}\right\}$ with sampling rate $f_{\mathrm{s}}=1 / T_{\mathrm{s}} \geq 2 f_{2}$. The number of samples is equal to $N$, where $N$ is the integer part of $\tau_{i} f_{\mathrm{s}}$.

Each sample of the chirp-signal is given as follows:

$$
x_{\mathrm{r}}=x\left(r T_{\mathrm{s}}\right)=A \cos \left(2 \pi\left(\frac{\Delta f}{2 N} r+f_{1}\right) r T_{\mathrm{s}}+\varphi_{0}\right),
$$

where $a=\Delta f / 2 \tau_{i}, \Delta f=f_{2}-f_{1}$ is the deviation of the frequency, $f_{1}$ is the initial frequency, $f_{2}$ is the final frequency, $\tau_{i}$ is the duration of the chirp signal, $\varphi_{0}$ is the initial phase, $r=\overline{0, N-1}$.

The algorithm of the digital matching filter based on a convolution in the time domain can be shown as follows:

$$
y_{n}=\sum_{m=0}^{N-1} x_{n-m} h_{m} w_{m} .
$$

The IR of the matching filter without a smoothing window is a mirror reflection of the input signal (2):

$$
h_{n}=x_{N-n}, \quad \text { where } n=\overline{1, N} .
$$

Here, $y_{n}$ is the $n$-th convolution operation result; $\left\{x_{n}\right\}$ are the input signal samples; $\left\{h_{n}\right\}$ are the weight factors of the IR, $N$ is the number of weight factors as well as input signal samples, $\left\{w_{n}\right\}$ are the smoothing window samples. In order to reduce the effect of Gibbs' oscillations, the smoothing window $\left\{w_{n}\right\}$ is used $[1,3]$. The total number of convolutions is $2 N-1$. For a rectangular window, $w_{n}=1$ is assigned. As a result of the chirp signal matched filtering, both main lobe and side lobes are formed. Thus, a few central samples (results of the convolution operations) create the main lobe, whilst other samples form side lobes. According to algorithm (3), the total number of multiplications needed to obtain in one convolution is $N$. However, if MF consists of $N$ parallel channels containing multipliers, one convolution is carried out in the time of one multiplication interval, which does not exceed $T_{\mathrm{s}}$.

In papers [5-7] it had been demonstrated that the initial phase and frequency, as well as the sampling rate of both short LFM signals and matched filter impulse characteristics had been an important influence upon the compression of these signals within the time domain. It was demonstrated that they have an important impact on the adjustment of the LFM signal amplitude spectrum to the selected window. The results of this study show that the compression of chirp signals achieves the best results when the above parameters are optimally set, and in the filtration process a rectangular window is used with the simultaneous implementation of nonlinear operations on the convolutions. This selection is the basis of minimization of the variance of the spectrum in the frequency domain. This allows to obtain high compression while achieving the borderline time resolution expressed by the width of the main lobe - the main lobe has a width equal to one sampling period only. The achieved PSR is up to $20 \mathrm{~dB}$ better for the short LFM signals with $\mathrm{BT} \leq 100$ (while maintaining a minimum width of the main lobe) than when using classical analog and digital methods.

\section{Compression results of LFM signals with non-zero initial frequency}

Figure 1 shows sample filtration results of LFM signals with the same BT obtained in computer simulations: one with a zero and another one with non-zero initial frequency. In both cases a rectangular window and nonlinear operations were used, and the initial phases of the signals and their sampling rates were optimally matched.

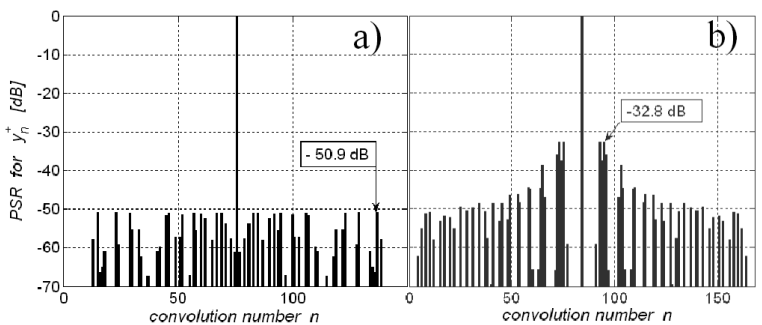

Fig. 1. The results of matched filtering with the use of a rectangular window and nonlinear operations at optimally matched sampling rate and an initial phase: (a) the chirp parameters: $f_{1}=0, \mathrm{BT}=37.5$; (b) the chirp parameters: $f_{1}=0.1 B, \mathrm{BT}=37.5$.

While using chirp signals with a non-zero frequency we always obtain a lower value of a PSR coefficient than while using signals with a zero initial frequency. Table shows the results of chirp signals compression at $15<\mathrm{BT}<300$ with the use of a rectangular window, nonlinear operations and optimally matched initial phases and sampling rate. Initial frequency is expressed as its percentage value for the chirp signal band. The results have been obtained using the methods of computer simulation. 
Results of chirp signals compression.

TABLE

\begin{tabular}{c|c|c|c|c|c|c|c|c}
\hline \hline \multirow{2}{*}{$\#$} & $f_{1} / B$ & \multicolumn{7}{|c}{ PSR [dB] } \\
\cline { 3 - 8 } & {$[\%]$} & $\mathrm{BT}=15$ & $\mathrm{BT}=30$ & $\mathrm{BT}=37.5$ & $\mathrm{BT}=60$ & $\mathrm{BT}=90$ & $\mathrm{BT}=105$ & $\mathrm{BT}=300$ \\
\hline 1 & 0 & 45.28 & 51.17 & 50.87 & 52.98 & 54.99 & 55.75 & 60.57 \\
2 & 1 & 46.68 & 48.08 & 48.53 & 51.64 & 49.58 & 52.16 & 52.47 \\
3 & 2 & 45.83 & 48.09 & 46.94 & 47.25 & 47.50 & 47.37 & 46.79 \\
4 & 3 & 42.66 & 43.82 & 42.76 & 44.57 & 43.98 & 43.98 & 42.15 \\
5 & 4 & 44.73 & 42.84 & 41.94 & 41.43 & 41.56 & 41.35 & 39.34 \\
6 & 5 & 42.68 & 40.45 & 40.92 & 40.17 & 39.32 & 38.83 & 37.73 \\
7 & 6 & 41.29 & 38.78 & 38.99 & 38.05 & 36.54 & 36.18 & 36.76 \\
8 & 7 & 38.62 & 38.27 & 36.45 & 35.37 & 34.28 & 34.15 & 36.15 \\
9 & 8 & 38.18 & 36.97 & 35.29 & 33.96 & 3390 & 33.38 & 35.35 \\
10 & 9 & 37.19 & 35.30 & 34.33 & 32.62 & 32.90 & 32.62 & 34.40 \\
11 & 10 & 35.93 & 33.32 & 32.80 & 32.66 & 32.53 & 32.75 & 33.40 \\
12 & 15 & 29.35 & 30.23 & 27.50 & 30.72 & 30.33 & 30.59 & 30.02 \\
13 & 20 & 28.95 & 28.80 & 27.42 & 28.47 & 28.19 & 28.05 & 27.65 \\
14 & 30 & 26.67 & 25.05 & 25.40 & 25.34 & 25.06 & 24.94 & 24.58 \\
15 & 40 & 23.31 & 23.26 & 23.26 & 22.72 & 23.01 & 23.06 & 23.12 \\
16 & 50 & 22.56 & 21.34 & 21.13 & 20.90 & 20.79 & 20.76 & 20.63 \\
17 & 60 & 20.33 & 20.17 & 20.27 & 20.22 & 20.31 & 20.35 & 19.82 \\
18 & 70 & 19.99 & 19.39 & 19.23 & 18.90 & 18.73 & 18.68 & 18.48 \\
19 & 80 & 19.88 & 19.07 & 18.33 & 18.33 & 17.86 & 17.09 & 16.87 \\
20 & 90 & 18.74 & 17.50 & 16.53 & 16.57 & 16.14 & 15.86 & 15.26 \\
21 & 95 & 17.31 & 16.98 & 16.05 & 16.27 & 15.64 & 15.26 & 14.80 \\
& & & & & & & & \\
& & & & & & & & \\
\end{tabular}

For every initial frequency an optimally matched initial phase of a chirp signal modified, at which maximal compression was obtained - an optimally matched initial phase generally increases with the increase of initial frequency. An optimally matched sampling rate still remains in the range of $f_{\mathrm{N}} \leq f_{\text {sopt }} \leq f_{N}+0.07 f_{N}$ (where $f_{N}$ - the Nyquist frequency). With the increase of the signal initial frequency, the impact of its initial phase on compression decreases. At zero initial frequency the initial phase shift of $1^{\circ}$ for an optimally matched phase results in an average decrease of PSR from 2 to $3 \mathrm{~dB}$. For the initial frequency higher or equal to $0.1 B$, the initial phase shift of $1^{\circ}$ for an optimally matched phase results in an average change of PSR in the range of $0.03 \mathrm{~dB}$ to $0.5 \mathrm{~dB}$.

The variance of the amplitude spectrum continues to grow with the increase of the initial frequency. Figure 2 shows the changes of PSR according to the initial frequency for the signals with different BT. The PSR coefficients were determined at optimally matched sampling rates and initial phases of signals, using the rectangular window and nonlinear operations in the process of filtration.

In general, while using chirp signals with a non-zero initial frequency a lower PSR is obtained, which systematically decreases with the increase of the initial frequency. A chirp signals spectrum with a non-zero initial frequency at an appropriately matched signal initial phase and an optimally matched sampling rate increasingly deviates

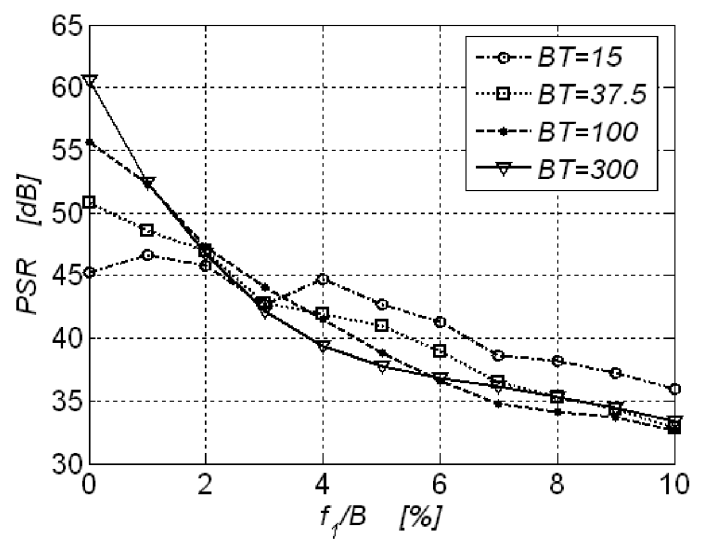

Fig. 2. The changes of PSR according to the initial frequency for the signals with different BT.

from rectangular (the variance of the amplitude spectrum increases) with the increase of the signal initial frequency.

Exceptions are chirp signals of $6 \leq \mathrm{BT}<20$, where at initial frequencies lower than $0.01 B$ a slight PSR increase (up to $2.5 \mathrm{~dB}$ ) is obtained. However, further increase of initial frequency results in the decrease of PSR. For chirp signals of BT $\geq 15$ with initial frequency $f_{1} \geq 0.1 B$ the maximal obtainable PSR practically does not depend on BT.

Existing sampling methods of band signals, whose spectrum is transferred by means of the carrier signal $f_{0}$ 
with a frequency significantly higher than $B\left(f_{0} \gg B\right)$ [2], may be effectively used for sampling signals considered in this study, which do not possess a carrier.

In this context, if a chirp signal has initial and final frequency respectively

$$
f_{1}=n B \quad \text { and } \quad f_{2}=(n+1) B,
$$

where $n=1,2, \ldots$ and a carrier signal frequency is replaced by central frequency $f_{m}=\frac{f_{1}+f_{2}}{2}$ then analogously to the paper [2] a relation will be obtained

$$
\frac{2 f_{m}-B}{k} \geq f_{\mathrm{s}} \geq \frac{2 f_{m}+B}{k+1},
$$

where $k$ is any natural number providing $f_{\mathrm{s}} \geq 2 B$, $B=f_{2}-f_{1}$.

Consequently, for chirp signals with a non-zero initial frequency according to Eq. (5), identical compressions will be obtained, with identical $f_{\mathrm{s}}$ as for chirp signals with a zero initial frequency and the identical band and duration. Such an approach is much simpler to implement than the one resulting from the direct use of Nyquist's frequency $f_{\mathrm{s}} \geq 2 B$. Moreover, it allows to receive maximal obtainable compressions.

\section{Conclusion}

The paper shows that for the LFM signals an initial frequency different from zero has a negative influence on their compression - the PSR coefficient systematically decreases with the increase of the initial frequency. While using existing methods of band signals sampling for the LFM signals with a non-zero initial frequency, it is possible to obtain identical results as for the LFM signals with a zero initial frequency and the identical band and duration. The initial frequency $f_{1}$ and the final frequency $f_{2}$ of an LFM signal must satisfy the condition: $f_{1}=n B$ and $f_{2}=(n+1) B, n=1,2, \ldots$

\section{References}

[1] J. Tsui, Digital Techniques for Wideband Receivers, Artech House, Boston 1995.

[2] R.G. Lyons, Understanding Digital Signal Processing, Addison Wesley Longman Inc., New York 1997.

[3] P. Tortoli, M. Baldanzi, F. Guidi, C. Atzeni, Microwaves Radio Freq. 5, 135 (1997).

[4] B.R. Mahafza, A.Z. Elsherbeni, MATLAB Simulations for Radar System Design, CRC Press LLC, Washington 2004.

[5] W. Pogribny, T. Leszczyński, Electron. Telecommun. Quarterly 54, 113 (2008).

[6] T. Leszczyński, Arch. Acoust. 37, 295 (2012).

[7] W. Pogribny, T. Leszczyński, Arch. Acoust. 36, 861 (2011). 